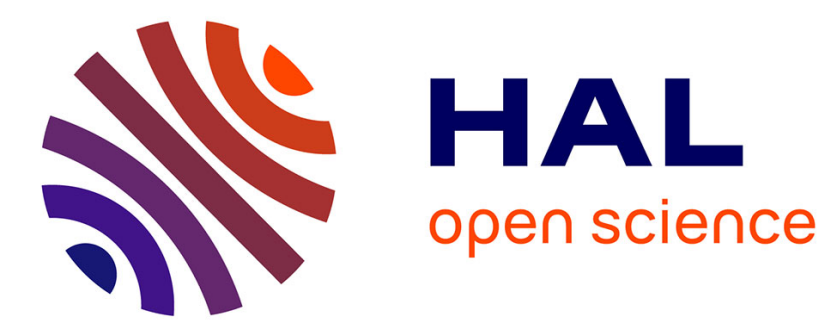

\title{
A system close to a threshold of instability
}

Elena Floriani, Dimitri Volchenkov, Ricardo Lima

\section{To cite this version:}

Elena Floriani, Dimitri Volchenkov, Ricardo Lima. A system close to a threshold of instability. Journal of Physics A: Mathematical and Theoretical, 2003, 36, pp.4771-4783. hal-00825720

\section{HAL Id: hal-00825720 \\ https://hal.science/hal-00825720}

Submitted on 24 May 2013

HAL is a multi-disciplinary open access archive for the deposit and dissemination of scientific research documents, whether they are published or not. The documents may come from teaching and research institutions in France or abroad, or from public or private research centers.
L'archive ouverte pluridisciplinaire HAL, est destinée au dépôt et à la diffusion de documents scientifiques de niveau recherche, publiés ou non, émanant des établissements d'enseignement et de recherche français ou étrangers, des laboratoires publics ou privés. 


\title{
A system close to a threshold of instability
}

\author{
E. Floriani ${ }^{1 *}$, D. Volchenkov ${ }^{2} \nmid$ R. Lima ${ }^{1}$ \\ ${ }^{1}$ Centre de Physique Théorique, UPR 7061 du CNRS case 907, \\ 13288 Marseille Cedex 09, France \\ ${ }^{2}$ BiBoS, University Bielefeld, Postfach 100131, \\ D-33501, Bielefeld, Germany
}

May 24, 2013

\begin{abstract}
We propose a probabilistic model for a system at a threshold of instability. The distribution of residence times below the threshold that characterizes the properties of the system is studied analytically in various cases.
\end{abstract}

PACS codes: $05.40 .+b, 05.40+j, 05.40 .-\mathrm{a}$

\section{Introduction}

Systems driven by random processes at a threshold of instability, may exhibit a random switching of a signal between a quiescent (stable) and a bursting (unstable) state. Such an

\footnotetext{
*E-mail: floriani@cpt.univ.mrs.fr

${ }^{\dagger}$ The Alexander von Humboldt Fellow, volchenk@physik.uni-bielefeld.de
} 
intermittent behavior is observed in a broad class of different systems in physics and nonlinear dynamics. Depending on its origin, the intermittent behavior either corresponds to the classification proposed by Pomeau-Manneville [1] (the I-III types intermittency) or shows the features characteristic of crisis-induced intermittency [2]. In both cases, the parameters of the models are static. Another example of intermittent behavior, called on-off intermittency, has been introduced in [3] and then observed numerically and experimentally [4]-[14]. The mechanism for this type of intermittency relies on a random forcing of a bifurcation parameter through a bifurcation point.

The ergodic properties of a system at a threshold of instability can be partially characterized by the distribution of the quiescent times (the durations of laminar phases) $P(t)$. Indeed, a complete characterization of the statistical properties of the system would imply the knowledge of residence times distribution in all the regions of phase space and not only in the laminar regions. However, the former is the first important statistical indicator of such a dynamics and this is the reason why we focus on the quiescent times distribution in the present study.

Depending on the particular type of intermittency exhibited by the system, the distribution $P(t)$ can display either an exponential or an inverse power law tail of exponent $\gamma$. In particular, the power law statistics of the quiescent times distribution is claimed to be typical for the systems showing the on-off type intermittency, and the value of exponent $\gamma$ depends in general on the nonlinearity characteristic of the dynamical system considered [13]. For example, in experiments on ion-acoustic instabilities in a laboratory plasma [14], due to nonlinear effects, the exponent of the power law depends on the value of a control parameter.

In the present paper, we investigate the effect produced on the statistics of laminar phases by 
the stochastic fluctuations of a system state variable (a bifurcation parameter) near the fluctuating threshold of instability (a bifurcation point). In our study, we do not refer to any definite physical system displaying an intermittent behavior. For the toy model which we introduce, the bifurcation parameter and the bifurcation point are considered as random independent variables. It is supposed that intermittency takes place in the system, when the process crosses the threshold value.

The control parameter of the system is the number $\eta \in[0,1]$, which represents a relative frequency of fluctuation of the threshold value: varying the parameter $\eta$ amounts to modifying the relation between the characteristic time scales of the threshold variable and of the state variable. At $\eta=0$ (when the state and the threshold varible have the same time scale) the statistics of laminar phases is exponential, while at $\eta=1$ (the limiting case of quenched threshold) it can be power law; for the intermediate values $0<\eta<1$, the statistics is mixed, becoming exponential for sufficiently large times.

In general, the statistics of laminar phases depends on the statistics of the random system state variable and threshold. We believe that the investigation of such a simple model could shed light on the dynamical origin of intermittent behavior occurring in more complex physical systems near a bifurcation point.

Let us notice that this model reproduces, in an extremely simplified manner, certain features of the dynamics of a weakly chaotic Hamiltonian system, with islands of stability in the phase space. The orbit of such a system (described by our state variable) typically stays for long times in the vicinity of a stability island until the moment when it crosses the separatrix (described by our threshold variable): the orbit then rapidly moves to other regions of phase space, until 
it reaches the neighborhood of another island, and the process starts again [15]. The evolution of the system is a succession of laminar phases, where diffusion dominates, and of flights; an important indicator of the statistical properties of the system is precisely the distribution of exit times from the neighborhood of an island of stability (corresponding to our $P(t)$ ).

The outline of the paper is as follows. In Sec. 2, we describe the model we introduce. In Sec. 3, we compute the distribution of residence times below the threshold with a generating functions approach. In many cases, the resulting distribution can be found explicitly. In Sec. 4, we consider the case of uniform statistics of the system state variable and threshold. Section 5 is devoted to conclusions.

\section{Description of the model}

Let us suppose that the state of a system can be characterized by a real number $x \in[0,1]$. Another real number $y \in[0,1]$ plays the role of a threshold of stability. The system is stable as long as $x<y$ and exhibits a sudden transition to the irregular state otherwise $(x \geq y)$.

We consider $x$ as a random variable distributed with respect to some given probability distribution function $\mathbb{P}\{x<u\}=F(u)$. In an analogous way, the value of the threshold $y$ is also a random variable distributed over the interval $[0,1]$ with respect to some other probability distribution function (pdf) $\mathbb{P}\{y<u\}=G(u)$. In general, $F$ and $G$ are two arbitrary leftcontinuous increasing functions satisfying the normalization conditions $F(0)=G(0)=0, F(1)=$ $G(1)=1$

Given a fixed real number $\eta \in[0,1]$, we define a discrete time random process in the following way. At time $t=0$, the variable $x$ is chosen with respect to $\operatorname{pdf} F$, and $y$ is chosen with respect 
to pdf $G$. If $x<y$, the process continues and goes to time $t=1$. At time $t \geq 1$, the following events happen:

i) with probability $\eta$, the random variable $x$ is chosen with pdf $F$ but the threshold $y$ keeps the value it had at time $t-1$. Otherwise,

ii) with probability $1-\eta$, the random variable $x$ is chosen with pdf $F$, and the threshold $y$ is chosen with pdf $G$.

If $x \geq y$, the process ends; if $x<y$, the process continues and goes to time $t+1$.

Eventually, at some time step $t$, when the state variable $x$ exceeds the threshold value $y$, the process stops, the system destabilizes, and the integer value $t=T$ acquired in this random process limits the duration of the laminar phase. The system then regains the stable state, when $x<y$, and the process starts again.

While studying the above model, we are interested in the distribution of the duration of laminar phases $P_{\eta}(T ; F, G)$ provided the probability distributions $F$ and $G$ are given and the control parameter $\eta$ is fixed.

Even if in our model the state variable $x$ is treated as a random variable, what is really important in what follows is the corresponding pdf $F$. It would be in fact possible to treat $x$ as a deterministic dynamical variable defined by the iterated images of a map of the interval $[0,1]$. In this case we would assume the existence of an invariant ergodic (Bernoulli) measure $d F$, for which $x$ is a generic orbit.

It is also to be noticed that the model proposed resembles closely to the coherent-noise models [16]-[17] discussed in connection with a standard sandpile model [18] in self-organized criticality, where the statistics of avalanche sizes and durations take power law forms. No exact 
analytical results concerning the coherent-noise models have been obtained so far. The model we propose has not been discussed in the literature before and, in principle, is much simpler than those discussed in [16]-[17], since it does not involve any spatial dynamics typical of such extended systems with quenched memory as the original sandpile models.

\section{Distribution of residence times below the threshold}

We are interested in the probability $P_{\eta}(T ; F, G)$ that the random process introduced in the previous section ends precisely at time $T$ with a crossing of the threshold, provided the distributions $F$ and $G$ are given and $\eta$ is fixed. We shall denote $P_{\eta}(T ; F, G)$ simply as $P(T)$. A straightforward computation shows directly from the definitions of Sec. 2 that

$$
P(0)=\int_{0}^{1} d G(y)(1-F(y))
$$

For $T \geq 1$, the system can either stay below the threshold in the laminar state ( a "survival") (S) or cross the threshold to a burst state (a "death") (D). Both events can take place either in the "correlated" way (with probability $\eta$; see i) in Sec. 2) (we denote them $S_{c}$ and $D_{c}$ ), or in the "uncorrelated" way (with probability $1-\eta$; see ii) in Sec. 2) $\left(S_{u}\right.$ and $\left.D_{u}\right)$. For $T=1$, we have for example:

$$
\begin{aligned}
P(1) & =P\left[S D_{c}\right]+P\left[S D_{u}\right] \\
& =\int_{0}^{1} d G(y) F(y) \eta(1-F(y)) \\
& +\int_{0}^{1} d G(y) F(y)(1-\eta) \int_{0}^{1} d G(z)(1-F(z))= \\
& =\eta B(1)+(1-\eta) A(1) B(0) .
\end{aligned}
$$


Similarly,

$$
P(2)=\eta^{2} B(2)+\eta(1-\eta) A(1) B(1)+\eta(1-\eta) A(2) B(0)+(1-\eta)^{2} A(1)^{2} B(0),
$$

where we define, for $n=0,1,2, \ldots$,

$$
A(n)=\int_{0}^{1} d G(y) F(y)^{n}
$$

and

$$
B(n)=\int_{0}^{1} d G(y) F(y)^{n}(1-F(y))=A(n)-A(n+1) .
$$

The general formula for $P(T)$, for all $T \geq 3$, is given in Appendix A.

It is useful to introduce the generating function of $P(T)$ :

$$
\hat{P}(s)=\sum_{T=0}^{\infty} s^{T} P(T)
$$

The generating property of the function $\hat{P}(s)$ is such that

$$
P(T)=\left.\frac{1}{T !} \frac{d^{T} \hat{P}(s)}{d s^{T}}\right|_{s=0} .
$$

Defining the following auxiliary functions

$$
\begin{gathered}
p(l)=\eta^{l} A(l+1), \quad \text { for } l \geq 1, \quad p(0)=0, \\
q(l)=(1-\eta)^{l} A(1)^{l-1}, \quad \text { for } l \geq 1, \quad q(0)=0, \\
r(l)=\eta^{l}[\eta B(l+1)+(1-\eta) A(l+1) B(0)], \quad \text { for } l \geq 1, \quad r(0)=0, \\
\rho=\eta B(1)+(1-\eta) A(1) B(0),
\end{gathered}
$$

we find

$$
\hat{P}(s)=B(0)+\rho s+\frac{s}{1-\hat{p}(s) \hat{q}(s)}[\hat{r}(s)+\rho \hat{p}(s) \hat{q}(s)+\rho A(1) \hat{q}(s)+A(1) \hat{q}(s) \hat{r}(s)],
$$


where $\hat{p}(s), \hat{q}(s), \hat{r}(s)$ are the generating functions of $p(l), q(l), r(l)$, respectively.

In the marginal cases $\eta=0$ and $\eta=1$, the probability $P(T)$ can be readily calculated. For $\eta=0$, equations (4), (5) give

$$
\hat{P}_{\eta=0}(s)=\frac{B(0)}{1-s A(1)}
$$

Applying the inverse formula (3) to equation (6), one gets

$$
P_{\eta=0}(T)=A(1)^{T} B(0)=\left[\int_{0}^{1} d G(y) F(y)\right]^{T} \int_{0}^{1} d G(y)(1-F(y))
$$

Therefore, in this case, for any choice of the pdf $F$ and $G$, the probability $P(T)$ decays exponentially.

For $\eta=1$, equations (4), (5) yield

$$
\hat{P}_{\eta=1}(s)=\hat{B}(s)
$$

so that,

$$
P_{\eta=1}(T)=B(T)=\int_{0}^{1} d G(y) F(y)^{T}(1-F(y))
$$

\subsection{Some examples of decay in the correlated case $\eta=1$}

We have just seen that, in the uncorrelated case $\eta=0$, the probability $P(T)$ decays exponentially for any choice of the pdf's $F$ and $G$.

In the correlated case $\eta=1$, many different types of behavior are possible, depending on the form of $F$ and $G$. We will examine an important class of pdf's, for which $P_{\eta=1}(T)$ can be explicitly computed from equation (7). We will take $F$ and $G$ absolutely continuous with respect 
to the Lebesgue measure, with

$$
\begin{gathered}
d F(u)=(1+\alpha) u^{\alpha} d u, \quad \alpha>-1, \\
d G(u)=(1+\beta)(1-u)^{\beta} d u, \quad \beta>-1 .
\end{gathered}
$$

Here we recognize the family of invariant measures of a map of the interval with a fixed neutral point [20].

Equation (7) gives in this case:

$$
P_{\eta=1}(T)=\frac{\Gamma(2+\beta) \Gamma(1+T(1+\alpha))}{\Gamma(2+\beta+T(1+\alpha))}-\frac{\Gamma(2+\beta) \Gamma(1+(T+1)(1+\alpha))}{\Gamma(2+\beta+(T+1)(1+\alpha))} .
$$

Using Stirling's approximation, we get for $T \gg 1$ :

$$
P_{\eta=1}(T)=\frac{(1+\beta) \Gamma(2+\beta)(1+\alpha)^{-1-\beta}}{T^{2+\beta}}\left(1+0\left(\frac{1}{T}\right)\right)
$$

For different values of $\beta$, the exponent of the threshold distribution, we get all possible (normalizable) power law decays of $P_{\eta=1}(T)$. Notice that the exponent $(-2-\beta)$ characterizing the decay of $P_{\eta=1}(T)$ is independent of the distribution $F$ of the state variable.

We were not able to prove that the asymptotic decay of $P_{\eta=1}(T)$ is algebraic for any choice of the distributions $F$ and $G$; nevertheless, we have not found any counterexample contradicting this conjecture. Let us consider in particular the case of $F$ uniform (the results of this section suggest in fact that what determines the decay of $P(T)$ is mostly the threshold pdf $G): P_{\eta=1}(T)$ is then a particular case of a Riemann-Liouville integral, and we did not find any case of non algebraic decay for large $T$ in the tables [19]. 


\subsection{Upper and lower bounds for $P(T)$ for any $\eta$}

We now compute bounds for $P(T)$, valid for any $\eta$ and any $T \geq 3$, starting from its expression given in Appendix A.

We will use the fact that

$$
A(1)^{n} \leq A(n) \leq A(1) \quad \text { and } \quad 0 \leq B(n) \leq A(1), \quad n=1,2, \ldots .
$$

The upper bound for $A(n)$ is trivial, since $0 \leq F(y) \leq 1$ for any $y \in[0,1]$. The lower bound is a consequence of Jensen's inequality, and of the fact that the function $x: \rightarrow x^{n}$ is convex on the interval $] 0,1[$ for any integer $n$.

We now replace these bounds for $A(n)$ and $B(n)$ in equations (17) to (20), and the resulting expressions in all the terms of the sum (16), except the one corresponding to the index $n=0$ in $P_{I}(T)$. (This term, which corresponds to a sequence of correlated survivals, has to be treated separately, in order not to lose information on the case $\eta=1$ ). Labeling by the index $k$ the number of uncorrelated survivals in the sequence of events considered in the sum (16), we get

$$
\begin{aligned}
P_{\eta}(T) & \leq\left[\eta^{T} B(T)+\eta^{T-1}(1-\eta) A(T) B(0)\right] \\
& +[\eta A(1)+(1-\eta) A(1) B(0)] \sum_{k=1}^{T-1} \gamma_{k}^{T-1}[(1-\eta) A(1)]^{k} \eta^{T-1-k}
\end{aligned}
$$

and

$$
\begin{aligned}
P_{\eta}(T) & \geq\left[\eta^{T} B(T)+\eta^{T-1}(1-\eta) A(T) B(0)\right] \\
& +(1-\eta) A(1) B(0) \sum_{k=1}^{T-1} \gamma_{k}^{T-1}[(1-\eta) A(1)]^{k}[\eta A(1)]^{T-1-k}
\end{aligned}
$$

where $\gamma_{k}^{T-1}$ represents the number of sequences of $T-1$ events $c, u(c=$ correlated survival, $u=$ 
uncorrelated survival) containing a number $k$ of events $u$, so that,

$$
\gamma_{k}^{T-1}=\left(\begin{array}{c}
T-1 \\
k
\end{array}\right)
$$

This implies the upper bound

$$
\begin{aligned}
P_{\eta}(T) & \leq \eta^{T} B(T)+(1-\eta) A(1) B(0)[\eta+(1-\eta) A(1)]^{T-1} \\
& +\eta A(1)\left\{[\eta+(1-\eta) A(1)]^{T-1}-\eta^{T-1}\right\}
\end{aligned}
$$

and the lower bound

$$
\begin{aligned}
P_{\eta}(T) & \geq \eta^{T} B(T)+(1-\eta) A(1)^{T} B(0) \\
& =\eta^{T} P_{\eta=1}(T)+(1-\eta) P_{\eta=0}(T) .
\end{aligned}
$$

We thus see that, for any $0 \leq \eta<1$, the decay of distribution $P(T)$ is bounded by exponentials. Furthermore, the bounds (9) and (10) are exact in the marginal cases $\eta=0$ and $\eta=1$.

\subsection{Behavior of $P(T)$ for intermediate times}

We have seen in section 3.1 that it exists a class of pdf's for which $P_{\eta}(T)$ decays like a power law when $\eta=1$. In section 3.2 , we show that for any $\eta \neq 1$ the asymptotic decay of $P_{\eta}(T)$ is exponential. We now make some remarks about the behavior of $P_{\eta}(T)$ for $\eta$ close to 1 .

The first thing to be noticed is that, for $T$ fixed, $P_{\eta}(T)$ is a continuous function of $\eta$, since it is a finite sum of continuous functions (see Appendix A). The results of section 3.2 imply of course that the continuity cannot be uniform in $T$. 
This means that, for any fixed interval of times $\left[T_{-}, T_{+}\right]$, with $T_{-}$in the range of validity of the power law asymptotics $(8)$ of $P_{1}(T), P_{\eta}(T)$ will be arbitrarily close to the same power law for $\eta$ sufficiently close to 1 .

For times $T \gg T_{+}$, the decay becomes exponential. We shall see in the next section that for the case of uniform densities, it is possible to estimate the value of the crossover time to the exponential behavior as a function of $\eta$.

\section{Distribution of quiescent times for the case of uniform densi- ties}

In this section, we consider the distribution of quiescent times for the special case of uniform densities $d F(u)=d G(u)=d u$ for all $u \in[0,1]$ and for any $\eta \in[0,1]$. In this case, simpler and explicit expressions can be given for $\hat{P}(s)$ and $P(T)$.

After some tedious but trivial computation, we get from equation (5):

$$
\hat{P}(s)=\frac{1}{1+(1-\eta) \gamma(s)}\left[\frac{1+\gamma(s)}{s}-\eta \gamma(s)\right]
$$

where $\gamma(s)$ is defined by

$$
\gamma(s)=\frac{\ln (1-\eta s)}{\eta s}
$$

The asymptotic behavior of $P(T)$ is determined by the singularity of the generating function $\hat{P}(s)$ that is closest to the origin.

For $\eta=0$, the generating function $\hat{P}(s)=(2-s)^{-1}$ has a simple pole, and therefore $P(T)$ decays exponentially, which agrees with the result of the previous section. In Figure 1, we have presented the distribution of quiescent times $P(T)$ in log-linear scale for $\eta=0$. 
For the intermediate values $0<\eta<1$, the generating function $\hat{P}(s)$ has two singularities. One pole, $s=s_{0}$, corresponds to the vanishing denominator $1+(1-\eta) \gamma(s)$, where $s_{0}=s_{0}(\eta)$ is the unique nontrivial solution of the equation

$$
-\ln (1-\eta s)=s \frac{\eta}{1-\eta}
$$

Another singularity, $s=s_{1}=\eta^{-1}$, corresponds to the vanishing argument of the logarithm. It is easy to see that $1<s_{0}<s_{1}$, so that the dominant singularity of $\hat{P}(s)$ is of polar type, and the corresponding decay of $P(T)$ is exponential, with rate $\ln \left(s_{0}(\eta)\right)$, for times much larger than the crossover time $T_{c}(\eta) \sim \ln \left(s_{0}(\eta)\right)^{-1}$.

The results of section 3.2 about the upper bound for the distribution $P(T)$ allow to be more precise about this decay rate. Equation (9) implies in particular (since $B(T) \leq A(1)$ ) that

$$
P(T) \leq[\eta A(1)+(1-\eta) A(1) B(0)][\eta+(1-\eta) A(1)]^{T-1},
$$

which in the case of uniform densities gives

$$
P(T) \leq \frac{1}{2}\left(\frac{1+\eta}{2}\right)^{T}
$$

We have then

$$
\frac{1}{s_{1}}=\eta<\frac{1}{s_{0}} \leq \frac{1+\eta}{2}
$$

and we see that the rate $\ln \left(s_{0}(\eta)\right)$ vanishes like $1-\eta$ as $\eta$ tends to 1 .

When $\eta$ tends to one, the two singularities $s_{0}$ and $s_{1}$ merge. More precisely, we have

$$
\hat{P}_{\eta=1}(s)=\frac{s+(1-s) \ln (1-s)}{s^{2}} .
$$


The corresponding dominant term in (13) is of order $\mathrm{O}\left(T^{-2}\right)$ [21]. This obviously agrees with the exact result one can get from equation (7), with $d F(u)=d G(u)=d u$ :

$$
P_{\eta=1}(T)=\frac{1}{(T+1)(T+2)}
$$

In Figure 2, we have drawn the distribution of quiescent times $P_{\eta=1}(T)$ that exhibits the powerlaw decay, with the slope $\gamma=-2$.

In the case of uniform densities, it is possible to get an expression of $P(T)$ for all times, and for any value of $\eta$, by applying the inversion formula (3) to equation (11). We have (see Appendix B):

$$
P(T)=\frac{\eta^{T}}{(T+1)(T+2)}+\sum_{k=1}^{T} \frac{\eta^{T}}{(T-k+1)(T-k+2) k} \sum_{m=1}^{k}\left(\frac{1-\eta}{\eta}\right)^{m} c(m, k),
$$

where $c(m, k)$ is defined by

$$
\begin{aligned}
& c(m, k)=m ! \quad \sum \\
& l_{1}+l_{2}+\cdots+l_{m}=k \\
& l_{i} \geq 1 \\
& \frac{l_{1} l_{2} \cdots l_{m-1} l_{m}}{\left(l_{1}+1\right)\left(k-l_{1}\right)\left(l_{2}+1\right)\left(k-l_{1}-l_{2}\right) \cdots\left(l_{m-1}+1\right)\left(k-l_{1}-l_{2}-\cdots-l_{m-1}\right)\left(l_{m}+1\right)} .
\end{aligned}
$$

When $\eta \neq 0$, there is an alternative way of writing the previous expression:

$$
P(T)=\frac{\eta^{T}}{(T+1)(T+2)}+\sum_{k=1}^{T} \frac{\eta^{T+1}}{(T-k+1)(T-k+2)} \sum_{l=1}^{\infty}(1-\eta)^{l} b(l, k),
$$

where $b(l, k)$ is defined by 


$$
b(l, k)=\sum_{\substack{i_{1}+i_{2}+\cdots+i_{l}=k \\ i_{j} \geq 0}} \frac{1}{\left(i_{1}+1\right)\left(i_{2}+1\right) \cdots\left(i_{l-1}+1\right)\left(i_{l}+1\right)} .
$$

In Figure 3, we have plotted the distribution of quiescent times $P_{\eta}(T)$ for the intermediate values $\eta=0.5, \eta=0.7, \eta=0.9$, together with the analytical result (15).

Notice that in Figures 1 and 3 (where $\eta \neq 1$ ), we only plot distributions $P(T)$ up to relatively short quiescent times $(T=16, T=25)$, since these times are already bigger than the crossover time $T_{c}(\eta) \sim 1 / \ln \left(s_{0}\right)$ to the exponential decay $\exp \left(-\ln \left(s_{0}\right) T\right)\left(s_{0}\right.$ defined by equation (12)). For much longer times, very few survivals are observed, and the statistics gets worse. Of course, $T_{c}(\eta)$ grows as the parameter $\eta$ tends to 1 , so that we have good statistics for longer and longer times (in Figure 2, for $\eta=1$, the plot is for quiescent times $20 \leq T \leq 2000$ ).

\section{Discussion and Conclusion}

In this paper, we have presented a model for a system at a threshold of instability. We assumed that the system loses stability when the parameter characterizing its state becomes larger than a threshold value. We have treated both the state parameter $x$ and the value of threshold $y$ as random variables distributed over the unit interval with respect to different distribution laws $F$ and $G$ respectively. The natural control parameter in our model is the probability $\eta$, which represents a relative frequency of threshold changes such that if $\eta=1$, the threshold value $y$ does not change during a laminar phase. In the opposite case, if $\eta=0$, at each time step, a new threshold value is taken with respect to the probability distribution function $G$. 
Varying $\eta \in[0,1]$, provided the distributions $F$ and $G$ are given, one can control the statistics of switching events (i.e. when the state parameter passes through the stability threshold).

For many distributions $F$ and $G$, the model can be solved analytically. In particular, we exhibit a class of threshold pdf's that allow to obtain any possible (normalizable) power law decay of the distribution of laminar phases, when $\eta=1$. Furthermore, we show that for any choice of the distributions $F$ and $G$ the distribution of laminar phases $P(T)$ decays exponentially at $\eta=0$, and that $P(T)$ is bounded above by a decresing exponential function of rate $\left[\eta+(1-\eta) \int_{0}^{1} d G(y) F(y)\right]$ for any $0<\eta<1$.

We have then studied the model for the special case of uniform densities $d F(u)=d G(u)=d u$. In this case the distribution of laminar phases decays as $T^{-2}$ at $\eta=1$. For the intermediate values of $\eta$, the decay is mixed: even if the asymptotic behavior is exponential, when $\eta$ is close to 1 the exponential regime is reached only for very large time lengths of order $(1-\eta)^{-1}$, while for intermediate but large times, the decay is polynomial.

A natural question arising in this context is the relationship between the ergodic invariants that quantify the dynamics of deterministic systems, for example the Lyapunov exponents, and the scaling laws studied in our paper. The corresponding question for models of selforganised criticality is certainly also pertinent since in that case a relation is known between the Lyapunov spectrum and the transport properties [22]. In our case, however, because of the dynamical character not only of the state variable but also of the threshold, some extension of the definition of the invariants would be needed, which is beyond the scope of the present paper. 


\section{Acknowledgements}

This work has been performed in connection with the international research project "The Sciences of Complexity: From Mathematics to Technology to a Sustainable World", Zentrum für Interdisziplinäre Forschung (ZIF), Universität Bielefeld (Germany). Authors acknowledge the financial support from the Zentrum für Interdisziplinäre Forschung (ZIF), Universität Bielefeld. One of the authors (D.V.) also benefits from a scholarship of the Alexander von Humboldt Foundation (Germany) that he gratefully acknowledges. One of the authors (E.F.) wishes to thank Bastien Fernandez and Olivier Agullo for fruitful discussions.

\section{Appendix A}

Here we present the general formula for $P(T)$, for $T \geq 3$. Let $S_{c(u)}^{l}$ denote the occurrence of $l$ consecutive "correlated" ("uncorrelated") survivals.

$$
\begin{aligned}
& P(T)=\quad \sum \quad \sum_{j=c, u} P\left[S S_{c}^{l_{1}} S_{u}^{l_{2}} \ldots S_{u}^{l_{2 n}} S_{c}^{l_{2 n+1}} D_{j}\right] \\
& n=0,1, \ldots,\left[\frac{T-2}{2}\right] \\
& l_{1}+l_{2}+\ldots+l_{2 n+1}=T-1, \quad l_{i} \geq 1 \\
& +\quad \sum \quad \sum_{j=c, u} P\left[S S_{c}^{l_{1}} S_{u}^{l_{2}} \ldots S_{c}^{l_{2 n-1}} S_{u}^{l_{2 n}} D_{j}\right] \\
& n=1, \ldots,\left[\frac{T-1}{2}\right] \\
& l_{1}+l_{2}+\ldots+l_{2 n}=T-1, \quad l_{i} \geq 1 \\
& +\quad \sum_{j=c, u} P\left[S S_{u}^{l_{1}} S_{c}^{l_{2}} \ldots S_{c}^{l_{2 n}} S_{u}^{l_{2 n+1}} D_{j}\right] \\
& n=0,1, \ldots,\left[\frac{T-2}{2}\right] \\
& l_{1}+l_{2}+\ldots+l_{2 n+1}=T-1, \quad l_{i} \geq 1
\end{aligned}
$$




$$
\begin{gathered}
+\sum_{j=c, u} P\left[S S_{u}^{l_{1}} S_{c}^{l_{2}} \ldots S_{u}^{l_{2 n-1}} S_{c}^{l_{2 n}} D_{j}\right] \\
l_{1}+l_{2}+\ldots+l_{2 n}=T-1, \quad l_{i} \geq 1 \\
=P_{I}(T)+P_{I I}(T)+P_{I I I}(T)+P_{I V}(T),
\end{gathered}
$$

in which

$$
\begin{aligned}
& \sum_{j=c, u} P\left[S S_{c}^{l_{1}} S_{u}^{l_{2}} \ldots S_{u}^{l_{2 n}} S_{c}^{l_{2 n+1}} D_{j}\right]=\eta^{l_{1}+l_{3}+l_{5}+\ldots+l_{2 n+1}}(1-\eta)^{l_{2}+l_{4}+\ldots+l_{2 n}} \\
& \times A\left(l_{1}+1\right) A^{l_{2}-1}(1) A\left(l_{3}+1\right) A^{l_{4}-1}(1) \ldots A\left(l_{2 n-1}+1\right) A^{l_{2 n}-1}(1) \\
& \times\left[\eta B\left(l_{2 n+1}+1\right)+(1-\eta) A\left(l_{2 n+1}+1\right) B(0)\right], \\
& \sum_{j=c, u} P\left[S S_{c}^{l_{1}} S_{u}^{l_{2}} \ldots S_{c}^{l_{2 n-1}} S_{u}^{l_{2 n}} D_{j}\right]=\eta^{l_{1}+l_{3}+l_{5}+\ldots+l_{2 n-1}}(1-\eta)^{l_{2}+l_{4}+\ldots+l_{2 n}} \\
& \times A\left(l_{1}+1\right) A^{l_{2}-1}(1) A\left(l_{3}+1\right) A^{l_{4}-1}(1) \ldots A\left(l_{2 n-1}+1\right) A^{l_{2 n}-1}(1) \\
& \times[\eta B(1)+(1-\eta) A(1) B(0)], \\
& \sum_{j=c, u} P\left[S S_{u}^{l_{1}} S_{c}^{l_{2}} \ldots S_{c}^{l_{2 n}} S_{u}^{l_{2 n+1}} D_{j}\right]=\eta^{l_{2}+l_{4}+\ldots+l_{2 n}}(1-\eta)^{l_{1}+l_{3}+\ldots+l_{2 n+1}} \\
& \times A(1) A^{l_{1}-1}(1) A\left(l_{2}+1\right) A^{l_{3}-1}(1) \ldots A^{l_{2 n-1}-1}(1) A\left(l_{2 n}+1\right) A^{l_{2 n+1}-1}(1) \\
& \times[\eta B(1)+(1-\eta) A(1) B(0)], \\
& \sum_{j=c, u} P\left[S S_{u}^{l_{1}} S_{c}^{l_{2}} \ldots S_{u}^{l_{2 n-1}} S_{c}^{l_{2 n}} D_{j}\right]=\eta^{l_{2}+l_{4}+\ldots+l_{2 n}}(1-\eta)^{l_{1}+l_{3}+\ldots+l_{2 n-1}} \\
& \times A(1) A^{l_{1}-1}(1) A\left(l_{2}+1\right) A^{l_{3}-1}(1) \ldots A^{l_{2 n-3}-1}(1) A\left(l_{2 n-2}+1\right) A^{l_{2 n-1}-1}(1) \\
& \times\left[\eta B\left(l_{2 n}+1\right)+(1-\eta) A\left(l_{2 n}+1\right) B(0)\right]
\end{aligned}
$$


where we have used the notations (1) and (2). Furthermore, using the convolution formula of $n$ functions $h_{1}, \ldots, h_{n}$ at a point $M$ defined as

$$
\begin{aligned}
& \left(h_{1} * h_{2} * \ldots * h_{n}\right)(M)=\quad \sum \quad h_{1}\left(l_{1}\right) h_{2}\left(l_{2}\right) \ldots h_{n}\left(l_{n}\right) \\
& l_{1}+l_{2}+\ldots+l_{n}=M \text {, } \\
& l_{i} \geq 0
\end{aligned}
$$

and the definitions (4), one can rewrite $P(T)$ as a sum of convolutions. For $T \geq 3$, the terms $P_{I, I I, I I I, I V}(T)$ read:

$$
\begin{gathered}
P_{I}(T)=\sum_{n=0}^{\infty}\left(h_{1}^{I} * h_{2}^{I} * \ldots * h_{2 n+1}^{I}\right)(T-1), \\
P_{I I}(T)=\rho \sum_{n=1}^{\infty}\left(h_{1}^{I I} * h_{2}^{I I} * \ldots * h_{2 n}^{I I}\right)(T-1), \\
P_{I I I}(T)=\rho A(1) \sum_{n=0}^{\infty}\left(h_{1}^{I I I} * h_{2}^{I I I} * \ldots * h_{2 n+1}^{I I I}\right)(T-1), \\
P_{I V}(T)=A(1) \sum_{n=1}^{\infty}\left(h_{1}^{I V} * h_{2}^{I V} * \ldots * h_{2 n}^{I V}\right)(T-1),
\end{gathered}
$$

where we have introduced

$$
\begin{gathered}
h_{1}^{I, I I}(l)=h_{3}^{I, I I}(l)=\ldots=h_{2 n-1}^{I, I I}(l)=p(l), \\
h_{2}^{I, I I}(l)=h_{4}^{I, I I}(l)=\ldots=h_{2 n}^{I, I I}(l)=p(l), \\
h_{2 n+1}^{I}(l)=r(l), \quad h_{2 n}^{I V}(l)=r(l), \\
h_{1}^{I I I, I V}(l)=h_{3}^{I I I, I V}(l)=\ldots=h_{2 n-1}^{I I I, I V}(l)=q(l), \\
h_{2}^{I I I, I V}(l)=h_{4}^{I I I, I V}(l)=\ldots=h_{2 n-2}^{I I I, I V}(l)=p(l), \\
h_{2 n}^{I I I}(l)=p(l), \quad h_{2 n+1}^{I I I}(l)=q(l) .
\end{gathered}
$$


Using the property of generating functions

$$
\sum_{M=0}^{\infty} s^{M}\left(h_{1} * h_{2} * \ldots * h_{n}\right)(M)=\hat{h}_{1}(s) \hat{h}_{2}(s) \ldots \hat{h}_{n}(s)
$$

we get expression (5).

\section{Appendix B}

The following relations hold:

$$
\begin{aligned}
& \left.\frac{d^{n} \gamma(s)}{d s^{n}}\right|_{s=0}=-\frac{n !}{n+1} \eta^{n} \\
& \left.\frac{d^{n}}{d s^{n}} \frac{1+\gamma(s)}{s}\right|_{s=0}=-\frac{n !}{n+2} \eta^{n+1} \\
& \frac{d^{n}}{d s^{n}} f(s) g(s)=\sum_{k=0}^{n}\left(\begin{array}{c}
n \\
k
\end{array}\right) \frac{d^{k} f(s)}{d s^{k}} \frac{d^{n-k} g(s)}{d s^{n-k}}, \\
& \frac{d^{n}}{d s^{n}} \frac{1}{1-f(s)}=\frac{f^{(n)}(s)}{[1-f(s)]^{2}}+(n-1) ! \sum_{m=2}^{n} \frac{m !}{[1-f(s)]^{m+1}} \quad \sum \\
& l_{1}+l_{2}+\cdots+l_{m}=n \\
& l_{i} \geq 1 \\
& \frac{f^{\left(l_{1}\right)}(s) f^{\left(l_{2}\right)}(s) \cdots f^{\left(l_{m-1}\right)}(s) f^{\left(l_{m}\right)}(s)}{\left(l_{1}-1\right) !\left(n-l_{1}\right)\left(l_{2}-1\right) !\left(n-l_{1}-l_{2}\right) \cdots\left(l_{m-1}-1\right) !\left(n-l_{1}-l_{2}-\cdots-l_{m-1}\right)\left(l_{m}-1\right) !},
\end{aligned}
$$

where equation (21) is valid for all $n \geq 1$. 


\section{References}

[1] Y. Pomeau and P. Manneville, Commun. Math. Phys. 74, 1889 (1980).

[2] C. Grebogi, E. Ott, and J. A. Yorke, Phys. Rev. Lett. 50, 93 (1983).

[3] N. Platt, E. A. Spiegel, and C. Tresser, Phys. Rev. Lett. 70, 279 (1993).

[4] A. S. Pikovsky, Z. Phys. B 55, 149 (1984).

[5] L. Yu, E. Ott, Q. Chen, Phys. Rev. Lett. 65, 2935 (1990).

[6] S. C. Venkataramani, T. M. Antonsen Jr., E. Ott, J. C. Sommerer, Physica D 96, 66 (1996), Phys. Lett. A 207, 173 (1995).

[7] P. W. Hammer, N. Platt, S. M. Hammel, J. F. Heagy, B. D. Lee, Phys. Rev. Lett. 73, 1095 (1994).

[8] G. Zumofen, J. Klafter, Physica D 69, 436 (1993).

[9] J. Redondo, E. Roldán, G. J. de Valcárcel, Phys. Lett. A 210, 301 (1996).

[10] J. F. Heagy, N. Platt, S. M. Hammel, Phys. Rev. E 49, 1140 (1994).

[11] N. Platt, S. M. Hammel, J. F. Heagy phys. Rev. Lett. 72, 3498 (1994).

[12] Z. Qu, F. Xie, G. Hu, Phys. Rev. E 53, R1301 (1996).

[13] H. L. Yang, Z. Q. Huang, E. J. Ding, Phys. Rev. E 54, 3531 (1996).

[14] Th. Pierre, H. Klostermann, E. Floriani, R. Lima, Phys. Rev. E 62, 7241 (2000).

[15] J. D. Meiss, Phys. Rev. A 34, 2375 (1986). 
[16] M. E. J. Newman, K. Sneppen, Phys. Rev. E 54, 6226 (1996).

[17] K. Sneppen, M. E. J. Newman, Physica D 110, 209 (1997).

[18] P. Bak, C. Tang, K. Wiesenfeld, Phys. Rev. Lett. 59, 381 (1987).

[19] A. Erdelyi, W. Magnus, F. Oberhettinger, F. G. Tricomi, Tables of integral transforms, Volume II, McGraw-Hill (1954).

[20] X. J. Wang, Phys. Rev. A 40, 6647 (1989).

[21] Flajolet and Sedgewick, Analytic Combinatorics (preliminary version), chapter 5 (1993): INRIA Res. Rep. n. 2026 (http://algo.inria.fr/flajolet/Publications/books.html).

[22] Ph. Blanchard, B. Cessac, T. Kruger, J. Stat. Phys 98, 375 (2000). 


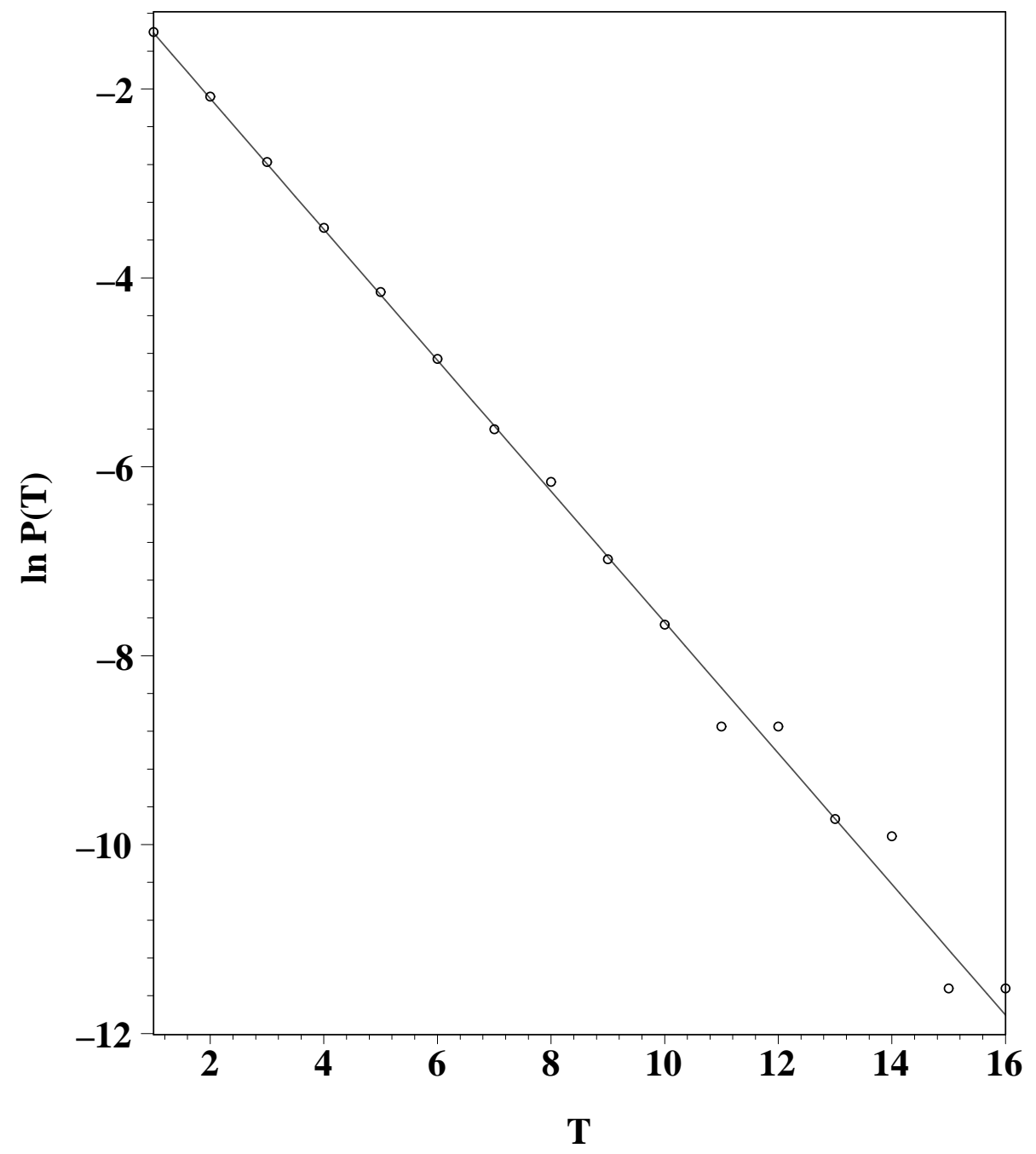

Figure 1: Distribution of quiescent times for the uniformly distributed variables $x$ and $y . P_{\eta}(T)$ decays exponentially for $\eta=0$, consistently with the analytical result $P(T)=2^{-(T+1)}$ (solid line). 


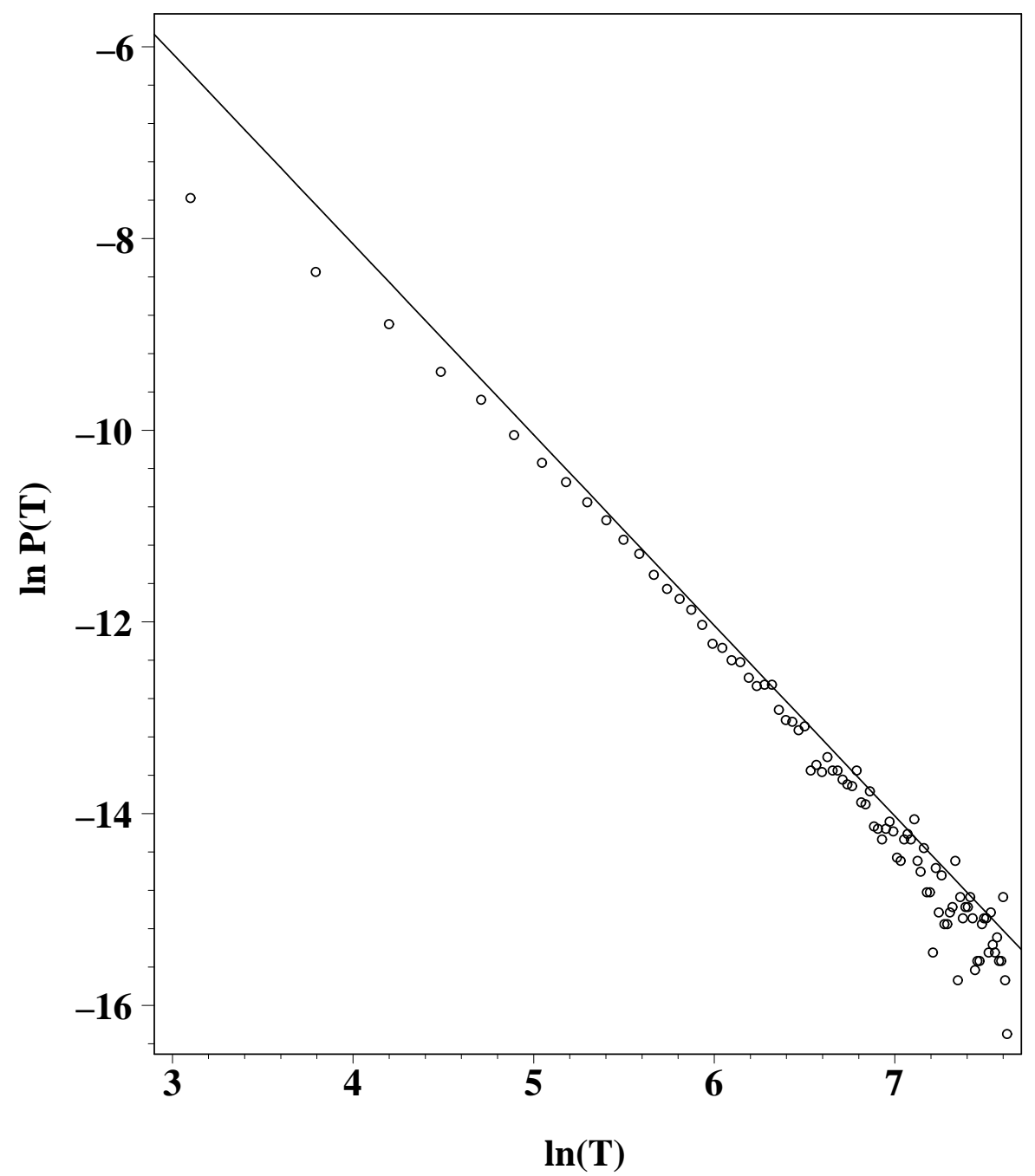

Figure 2: Distribution of quiescent times for the uniformly distributed variables $x$ and $y$. We show the power-law decay of $P_{\eta=1}(T)$ plotted in log-log scale. The solid line is given by Eq.(14). 


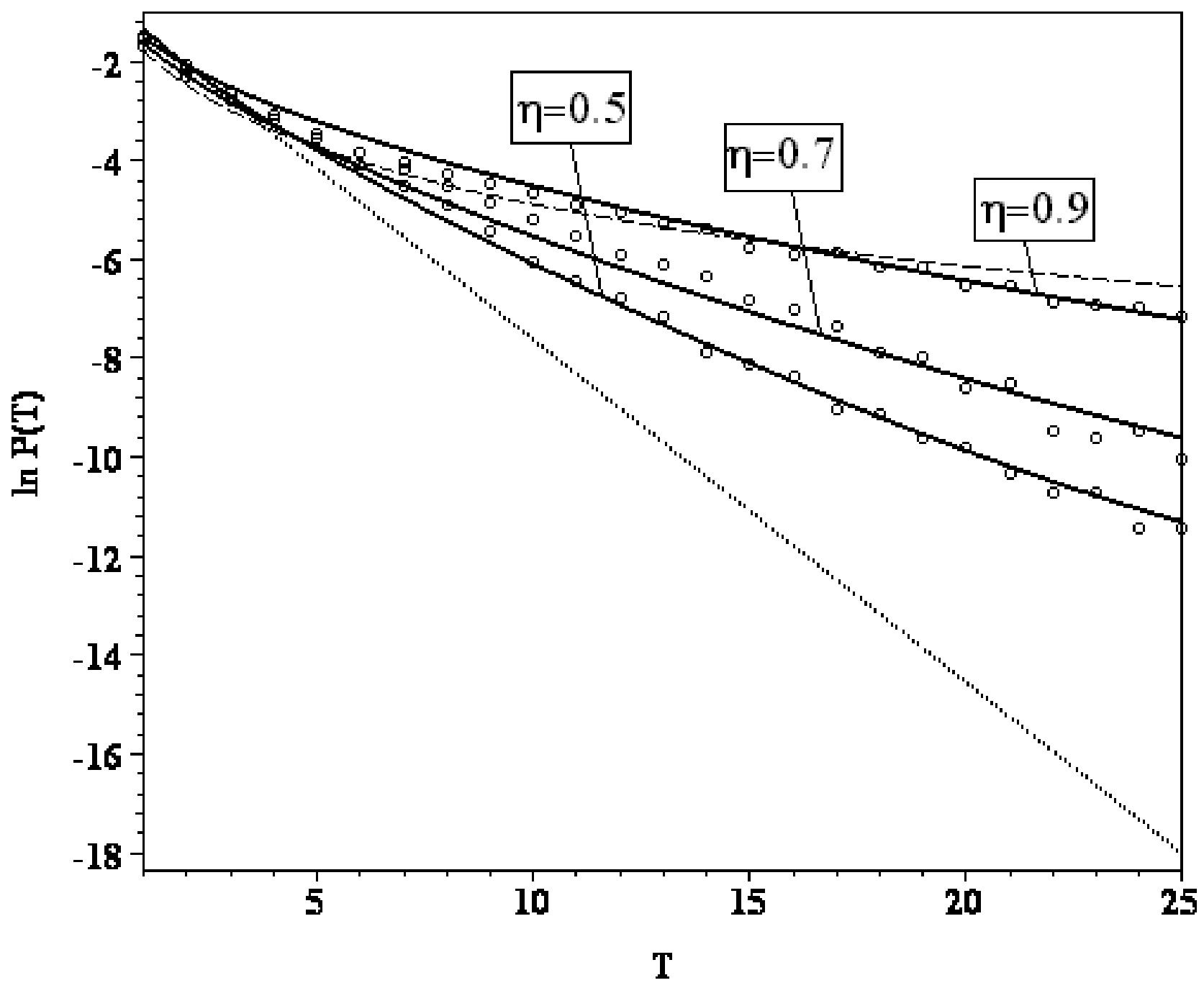

Figure 3: Distribution of quiescent times for the uniformly distributed variables $x, y$ at the intermediate values $\eta=0.5, \eta=0.7, \eta=0.9$ (circles). For comparison, the dotted line $2^{-T-1}$ presents the exponential decay for $\eta=0$, and the dashed line corresponds to $[(T+1)(T+2)]^{-1}$ for $\eta=1$ (Eq.(14)). The solid lines are given by Eq.(15) for $\eta=0.5, \eta=0.7, \eta=0.9$. 